\title{
High-pressure micro-discharges in etching and deposition applications
}

\author{
R Mohan Sankaran and K P Giapis ${ }^{1}$ \\ Division of Chemistry and Chemical Engineering, California Institute of Technology, \\ Pasadena, CA 91125, USA
}

Received 17 March 2003

Published 19 November 2003

Online at stacks.iop.org/JPhysD/36/2914

\begin{abstract}
High-pressure micro-discharges are promising sources of light, ions, and radicals and offer some advantages in materials processing applications as compared to other more conventional discharges. We review here results from etching experiments using stencil masks where the discharge is formed only in the pattern cutout. The mask consists of a thin metal-dielectric structure and is pressed against a Si wafer, which becomes part of the electric circuit. Pattern transfer takes place, albeit the profile shape appears to be limited by the expansion of the plasma into the etched hole at long etch times. We also review experiments on using micro-discharges as sources of radicals for materials deposition applications. In the latter case, the micro-discharges form in metal capillary tubes permitting incorporation of gas flow and a short reaction zone that can be controlled to favour production of specific radicals. We demonstrate these concepts by using $\mathrm{CH}_{4} / \mathrm{H}_{2}$ chemistry for diamond deposition on a heated Mo substrate. Good quality micro- and nano-diamond crystals could be produced.
\end{abstract}

\section{Introduction}

Micro-hollow cathode discharges or micro-discharges are direct current, high-pressure plasmas formed between two metal foils (a cathode with a pin-hole and an anode of arbitrary shape) separated by a thin layer of a dielectric material [1]. Compared to conventional glow discharges between parallel plates, these miniature discharges are capable of operating at a lower voltage drop but much higher current density [2]. The ensuing increased ionization has been attributed to the hollow electrode geometry which produces strong radial electric fields that result in oscillation of electrons in the cathode (Pendel effect [3]). Reducing the hole size to below $200 \mu \mathrm{m}$ in diameter permits stable operation at pressures exceeding atmospheric in a variety of atomic and molecular gases $[4,5]$. Optical studies have shown that these discharges contain highly excited states even at high-pressures ( $>100$ Torr) including ions, lying more than $50 \mathrm{eV}$ above ground state [6], and excimer dimers [7, 8]. While attractive for lighting applications, the presence of a large concentration of high-energy electrons in these microdischarges could be of interest for materials processing. In particular, the production of reactive radicals on a small spatial scale suggests the use of these plasmas as micro-reactors. In

1 Author to whom correspondence should be addressed. this paper, we discuss two representative materials applications of micro-discharges: silicon etching and diamond deposition.

\section{Silicon etching using patterned micro-discharges}

Plasma etching of silicon is a well-established process used extensively in the semiconductor industry to define sub-micron features with a high degree of anisotropy. It is also successfully used in patterning larger scale features for optical and MEMS applications. Pattern transfer generally requires multiple lithographic and etching steps that, in turn, are time consuming and costly as they rely on significant infrastructure. Moreover, there is a lot of waste associated with the use of bulk plasmas in patterning. For example, only a small fraction of the ions and reactive species produced in the bulk plasma contribute to the patterning process. Since the electron Debye length under typical low-pressure plasma conditions ( $<20$ mTorr) is on the order of hundreds of microns, the plasma remains outside the features being patterned. A shift in paradigm to the lithography and etching approach would be to use a stencil mask with the pattern already defined in it, then strike a plasma within the pattern in contact with the wafer surface. If the stencil mask could be used multiple times, it could lead to a virtually 'maskless' direct patterning technique. Since the plasma is formed only where it is needed to generate 
the reactive radicals and ions, neither plasma volume nor power would be wasted. In order for the plasma to form in such confined spaces, the electron Debye length must be decreased which is easily accomplished by increasing the ambient gas pressure. However, stable plasma operation at high-pressures is generally difficult as very large electric fields must be established and maintained for efficient electron acceleration and the ensuing ionization phenomena that sustain the plasma. Micro-hollow cathode discharges have an inherent advantage from this perspective by means of the Pendel effect. Enhanced ionization within the confines of a mask cutout could facilitate pattern transfer. The question is then, how small a feature can be achieved and with what fidelity? It has been shown that discharges could be struck in features as small as tens of microns [9], a length scale appropriate for MEMS device fabrication [10]. Furthermore, the ability to form micro-discharges in flexible stencil masks could allow the patterning of curved surfaces such as cylinders and spheres, a task of considerable difficulty with conventional patterning techniques. We present here an account of our effort to pattern silicon wafers using micro-plasmas formed in flexible stencil masks and discuss limitations of the technique.

Direct patterning is possible if and only if the cutout pattern in the stencil mask can be filled with plasma. The cylindrical confinement in holes takes full advantage of the Pendel effect, which may not necessarily occur in lines resulting in lower electron densities and much weaker ionization. Our initial efforts were therefore focused on fabricating and testing multiple hole and line patterns in metal-dielectric-metal structures. Devices were constructed from thin copper foils (100 $\mu$ m thick, $99.995 \%$ pure) which were spin-coated with polyimide films as described in [11]. These materials were chosen for degradation resistance when exposed to fluorocarbon chemistry, as well as for their flexibility. Holes were drilled and slots were cut out mechanically in the layered structure to produce a desired pattern. Either a solid copper foil or, in the case of etch experiments, a blanket n-type $\mathrm{Si}$ (100) wafer was pressed firmly against the mask as illustrated in figure 1 . The assembly was then placed in a reactor chamber and evacuated to $1 \times 10^{-6}$ Torr. Silicon wafers were cleaned prior to etching by dipping in a dilute $\mathrm{HF}$ solution (1-5\% in $\mathrm{H}_{2} \mathrm{O}$ ) for 1 min and rinsing with de-ionized water.

Discharges were struck in both pure argon and etch gas mixtures at a variety of pressures and plasma parameters. Complete pattern filling by the discharge depended on pattern shape and operating conditions. In the case of an array of identical holes, discharges were initiated in each hole simultaneously and filled all holes uniformly. An example

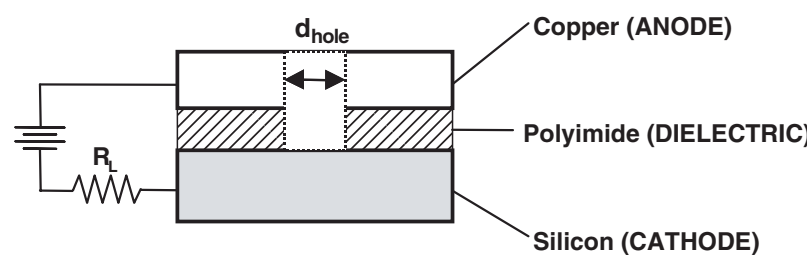

Figure 1. Schematic depiction of the micro-discharge set-up used for maskless etching of silicon. Current-limiting resistor, $R_{\mathrm{L}}=100 \mathrm{k} \Omega$. is shown in figure $2(a)$ for a $3 \times 3$ array of $200 \mu \mathrm{m}$ holes; the discharge was struck in a $\mathrm{CF}_{4} / \mathrm{Ar}$ mixture at 20 Torr. For line patterns, initial breakdown occurred in a part of the open structure requiring higher currents for complete space filling. Figure 2(b) shows a micro-discharge filling 3 lines $0.2 \mathrm{~mm} \times 10 \mathrm{~mm}$, operated in $\mathrm{CF}_{4} / \mathrm{Ar}$ at 20 Torr; the total current drawn by the discharge was $3 \mathrm{~mA}$. Although not shown here, more complex patterns consisting of multiple holes and crossed lines could also be filled with plasma, provided sufficient plasma current could be supplied.

The presence of reactive species in etch gas mixtures was verified by optical emission spectroscopy. The spectra were obtained by using standard optics, a $0.22 \mathrm{~m}$ SPEX 1680 double monochromator (gratings ruled with 1200 grooves $\mathrm{mm}^{-1}$, blazed at $630 \mathrm{~nm}$ ) and a Hamamatsu R928 photomultiplier tube. Figure 3 shows a representative spectrum of a $\mathrm{CF}_{4} / \mathrm{Ar}$ discharge $(1: 3)$ operated at 20 Torr and $1.5 \mathrm{~mA}$. Because of the large amount of argon in the mixture, only the most intense fluorine atom lines are visible at $685.6,690.2$, and $703.7 \mathrm{~nm}$ (marked by an asterisk). A spectrum for a $\mathrm{SF}_{6} / \mathrm{Ar}$ discharge operated at the same conditions is also shown for comparison. The latter discharge produces higher intensity atomic fluorine lines, corresponding to a larger concentration of these species. Thus, the two mixtures should etch silicon differently.
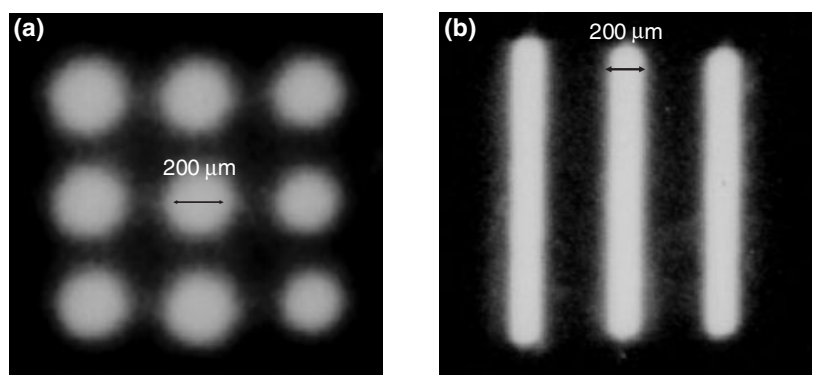

Figure 2. Photographs of $\mathrm{CF}_{4} / \mathrm{Ar}(1: 3)$ micro-discharge arrays at 20 Torr in copper-polyimide-copper devices for: $(a)$ a $3 \times 3$ array of $200 \mu \mathrm{m}$ holes (pitch $=400 \mu \mathrm{m}$ ), and $(b)$ an array of 3 lines each with width of $200 \mu \mathrm{m}$. Total discharge currents were $0.6 \mathrm{~mA}$ and $3 \mathrm{~mA}$, respectively.



Figure 3. Optical emission spectra of micro-discharges in copper-polyimide-copper structures operated at 20 Torr in (a) $\mathrm{CF}_{4} / \mathrm{Ar}(1: 3)$ and (b) $\mathrm{SF}_{6} / \mathrm{Ar}(1: 3)$. The discharge current was $0.2 \mathrm{~mA}$ in both cases. 
When a complete metal-dielectric-metal structure was used to pattern $\mathrm{Si}$ wafers, the etching was characterized by low rates due to deposition of sputtered cathode material on the $\mathrm{Si}$ surface. This observation suggests that either a low sputtering yield electrode must be used as the cathode or the Si wafer must become part of the circuit (the cathode itself). The latter configuration eliminated contamination from the electrode material and achieved high etch rates of silicon attributed to the direct contact between the discharge and the substrate. The etch rate depended on discharge current, pressure, and mixture composition. For example, a silicon etch rate of $7.7 \mu \mathrm{m} \mathrm{min}^{-1}$ was obtained when etching a single $200 \mu \mathrm{m}$ hole at 20 Torr using a mixture of $1: 3 \mathrm{CF}_{4} / \mathrm{Ar}$ discharge operated at 400 volts. After the discharge was initiated, the plasma voltage was kept constant for the duration of the experiment. It was found that etching of the substrate altered the shape of the cathode, which affected both the operating voltage and current. When the voltage was kept constant, the discharge current increased linearly with etch time.

Although we are discussing applications of high-pressure micro-discharges, etching experiments were confined to below 50 Torr. It was found that etching at higher pressures generally results in isotropic profiles with significant undercutting. Most of the experiments were performed between 10-50 Torr for high etch rates and profiles that conform to the mask at least initially (vide infra). Note that these pressures are still very high as compared to those employed in modern plasma etching reactors $(\sim 10$ mTorr $)$.

Scanning electron microscope (SEM) images of etched substrates were taken to determine etch rates and profiles. Parts $(a)-(c)$ of figure 4 show profiles obtained after etching in $\mathrm{CF}_{4} / \mathrm{Ar}(1: 3)$ discharges for $10 \mathrm{~min}, 15 \mathrm{~min}$, and $20 \mathrm{~min}$, respectively. In each case, the pressure was controlled at 20 Torr while the voltage drop between anode and cathode was
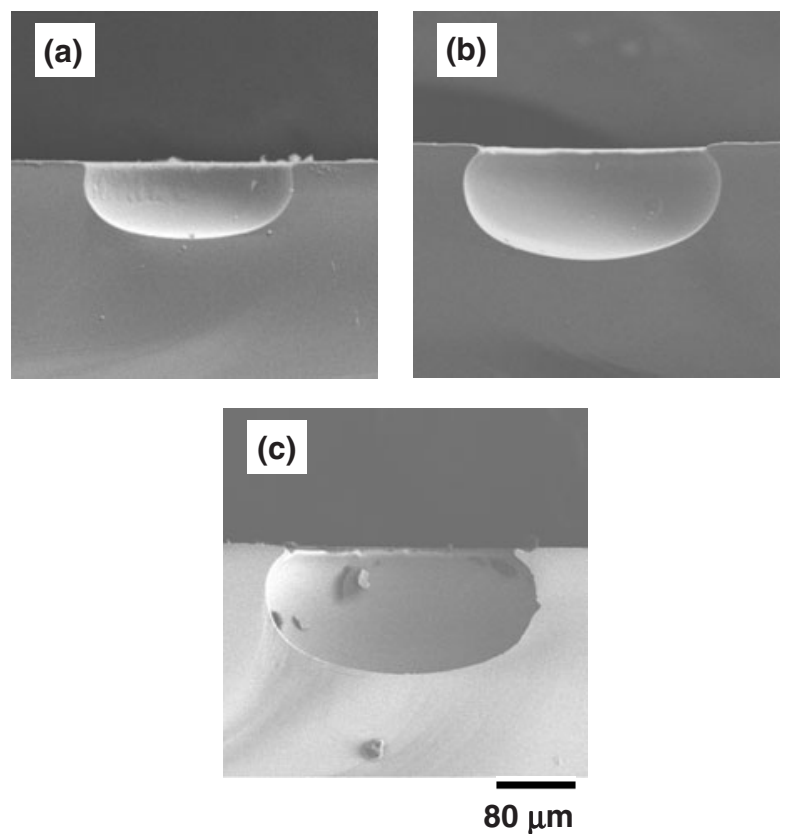

Figure 4. SEM images of single holes etched in silicon using $\mathrm{CF}_{4} / \mathrm{Ar}(1: 3)$ micro-discharges at 20 Torr for $(a) 10,(b) 15$, and (c) $20 \mathrm{~min}$. The stencil mask consisted of copper-polyimide layers with a single $200 \mu \mathrm{m}$ diameter hole. maintained at $400 \mathrm{~V}$. The same stencil mask with a $200 \mu \mathrm{m}$ diameter hole was used to etch each of these samples with no observable damage to the mask. At the early stages of etching (figure $4(a)$ ), the pattern transferred with good fidelity and virtually no dimensional loss; it was noted that the bottom of the profile was rounded. As etching continued (figure $4(b)$ ), erosion of the sidewalls occurred resulting in a bowl-shaped profile. At even longer times (figure 4(c)), the etch rate in the vertical direction diminished greatly while etching in the horizontal direction accelerated. For the etch times studied, there was very little undercutting of the mask. It is hypothesized that the bowl-shaped profile is a result of the expansion of the plasma into the etched void. As the plasma fills this volume, the sheath may conform to the shape of the generated cavity. A conformal sheath could result in ion bombardment of the cavity walls in a spatially uniform fashion, yielding etching of both the bottom and the sidewalls. Similar profiles were reported in sub-millimeter hollow cathode discharges operated in $\mathrm{Ne}$, albeit due to sputtering. In that case, initially cylindrical holes approached a stable spherical profile [12].

Silicon etch profiles were also studied for $\mathrm{SF}_{6} / \mathrm{Ar}(1: 3)$ discharges as a function of time. At identical pressure and bias conditions, the $\mathrm{Si}$ etch rate was substantially larger than that for $\mathrm{CF}_{4}$ gas mixtures with notable similarities in the profile (bowl-shaped). However, the profile also exhibits significant undercutting resulting in dimensional loss and larger holes (figure 5). Both observations are consistent with formation of a large number of fluorine atoms in the $\mathrm{SF}_{6}$ micro-discharge. In these experiments, the $\mathrm{Si}$ etch rate is proportional to the total discharge current. By plotting the discharge current as a function of etch time we can appreciate the significant differences in $\mathrm{Si}$ etch rate between $\mathrm{CF}_{4}$ and $\mathrm{SF}_{6}$ mixtures (figure 6). The increase in current observed in both cases is concomitant with the expansion of the discharge into the generated cavity. Assuming that the discharge is uniform over the entire hole, it appears that the cavity surface areathat is, the effective cathode area-increases a lot faster in the case of $\mathrm{SF}_{6}$. The larger etch rate in the case of $\mathrm{SF}_{6}$ has been confirmed by SEM measurements. Thus, unlike conventional plasma etching, the discharge current offers a method to monitor the etch process in situ. This observation suggests that the profile shape can be tailored by altering the operating conditions during the same experiment. For example, in figure 7 , etching of a $\mathrm{Si}$ wafer was started in a $\mathrm{CF}_{4} / \mathrm{Ar}(1: 3)$ gas mixture at 20 Torr; the voltage drop across the discharge was fixed at $400 \mathrm{~V}$. The discharge current was observed to increase in a slightly superlinear fashion. After $10 \mathrm{~min}$, the pressure was reduced to 15 Torr, resulting in an immediate decrease in the discharge current as the plasma stabilized at the new pressure. Again, the discharge current was observed to increase as etching progressed. After 10 more minutes, the pressure was again reduced to 13 Torr and the experiment continued with similar observations. After a total time of $30 \mathrm{~min}$, etching was stopped and the sample was imaged, as illustrated in figure 8. In comparison to the etch results of figures 4 and 5, the profile was etched approximately to the same depth but with less widening of the hole. By reducing the pressure during etching, the plasma density was decreased which may have affected the etching mechanism in 

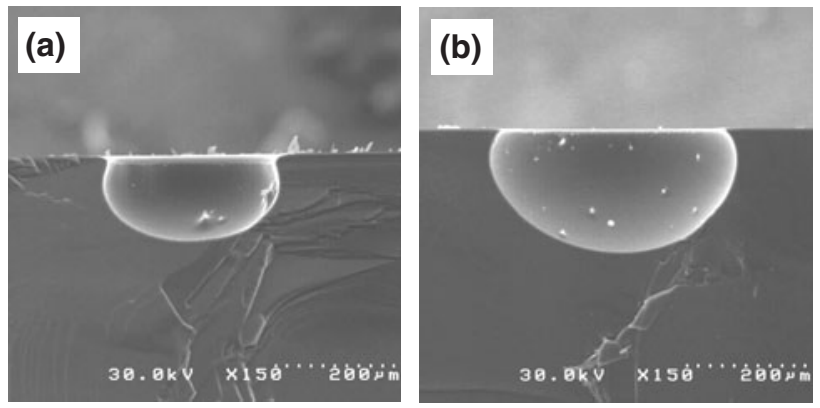

$200 \mu \mathrm{m}$

Figure 5. SEM images of single holes etched in silicon using $\mathrm{SF}_{6} / \mathrm{Ar}(1: 3)$ micro-discharges at 20 Torr in a structure with a $200 \mu \mathrm{m}$ diameter hole after $(a) 5$ and $(b) 10 \mathrm{~min}$.

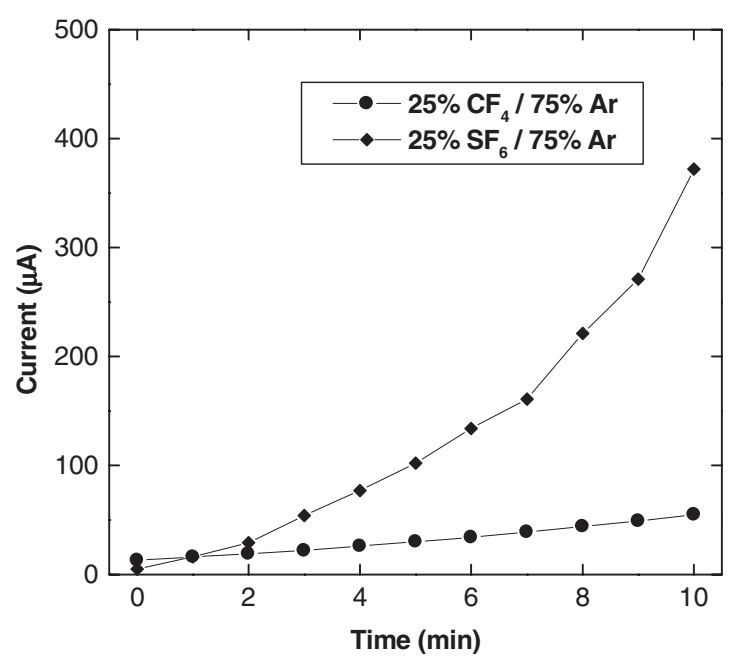

Figure 6. Discharge current versus time for etching a $200 \mu \mathrm{m}$ hole in silicon using $\mathrm{CF}_{4} / \mathrm{Ar}$ and $\mathrm{SF}_{6} / \mathrm{Ar}$ gas mixtures at 20 Torr. The discharge voltage was kept constant at $400 \mathrm{~V}$ in both cases.

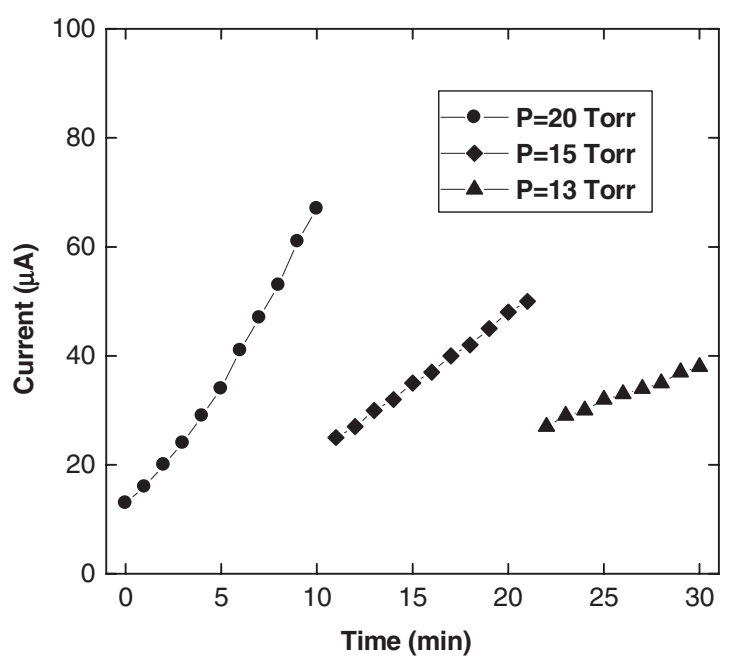

Figure 7. Discharge current as a function of time for etching a $200 \mu \mathrm{m}$ hole in silicon with pressure changes every $10 \mathrm{~min}$ during a total etch time of $30 \mathrm{~min}$. Other process conditions: $\mathrm{CF}_{4} / \mathrm{Ar}(1: 3)$, plasma potential $=400 \mathrm{~V}$.

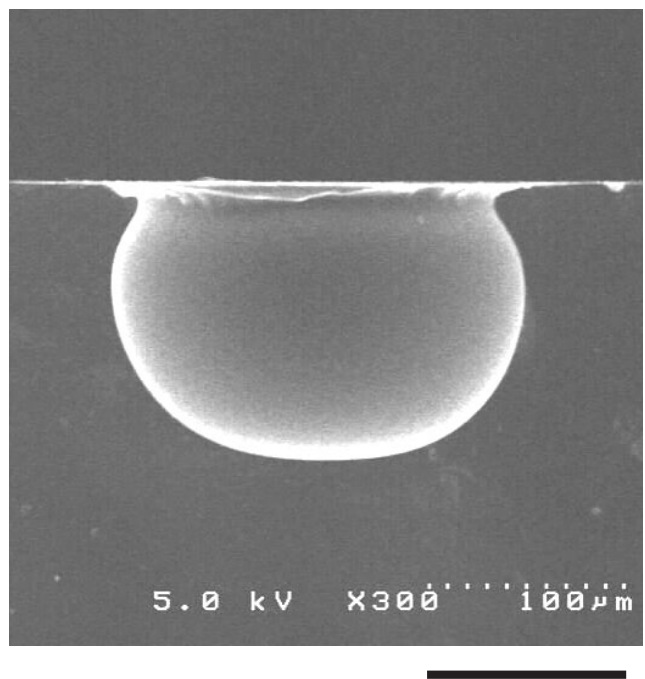

$100 \mu \mathrm{m}$

Figure 8. SEM image of single hole etched in silicon using the sequential pressure reduction process described in the text.
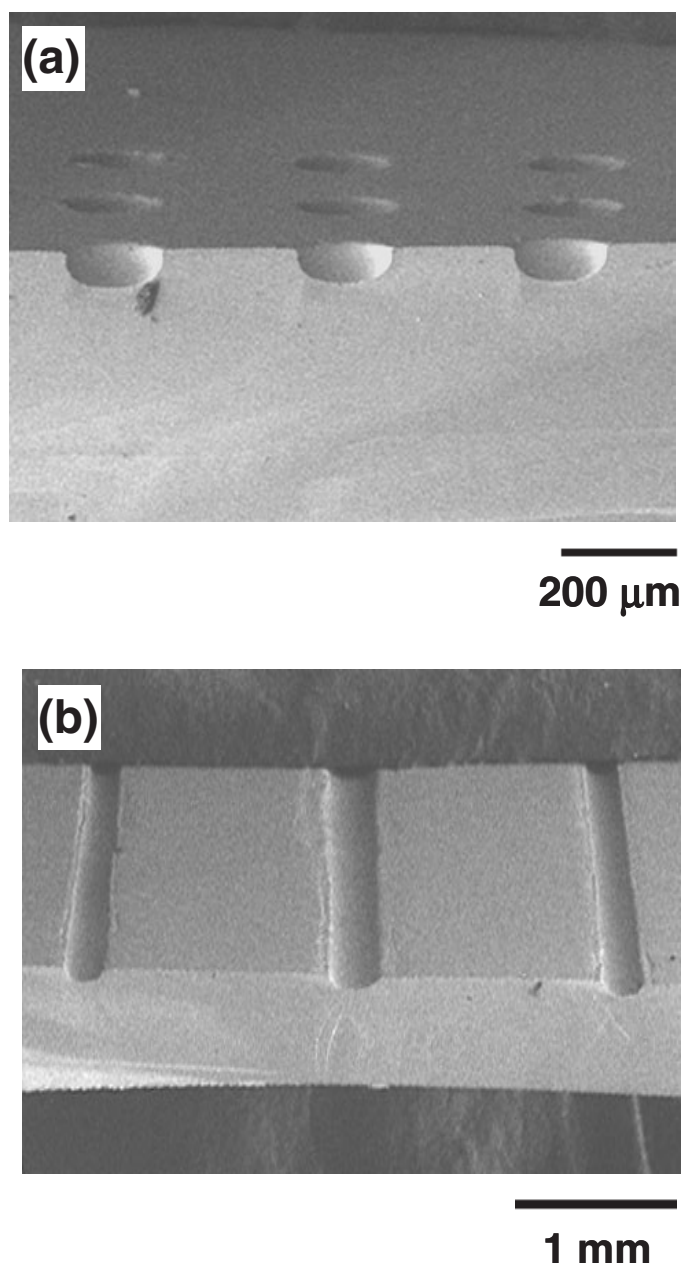

Figure 9. SEM images of silicon substrates after direct pattern transfer using a stencil mask with (a) $3 \times 3$ array of $200 \mu \mathrm{m}$ diameter holes and (b) 3 slots of $200 \mu \mathrm{m}$ width. Discharges were struck in $\mathrm{CF}_{4} / \mathrm{Ar}(1: 3)$ at 20 Torr and run for $10 \mathrm{~min}$ at constant plasma potential of $400 \mathrm{~V}$. 
the hole. Other changes in the plasma conditions may also have similar effects on the etch profile allowing etching of higher aspect ratio structures.

We have also etched arrays of $200 \mu \mathrm{m}$ holes simultaneously at the same process conditions as those used in singlehole experiments. For the same operating voltage, an array of $n$ holes required a discharge current approximately $n$ times larger than that for a single hole. Figure $9(a)$ shows a silicon wafer etched for 10 min using a stencil mask with an array of nine $200 \mu \mathrm{m}$ holes. Etching occurred uniformly in each hole and produced profiles nearly identical to those seen for individual holes. The ability to etch arrays of holes is especially attractive for fabrication of micro-discharge devices in silicon [6]. As a proof-of-concept, we have successfully initiated discharges in pure Ar after etching structures in silicon and evacuating the chamber from any reactive fluorine radicals. We have not yet studied what, if any, is the impact of the cathode shape on the characteristics of these discharges. While micro-discharges are normally operated in holes, we have also extended our patterning method to other shapes. Figure $9(b)$ illustrates a result for etching silicon (10 min in $1: 3 \mathrm{CF}_{4} / \mathrm{Ar}$ mixture, discharge current adjusted to fill the pattern) using a stencil mask with three parallel lines, each $200 \mu \mathrm{m}$ wide. There are notable similarities in cross-section with the arrays of holes.

\section{Micro-discharges in flow geometry: application to diamond deposition}

While the conventional planar (metal-dielectric-metal) structure of MHCDs offers advantages for lighting and etching applications, its use as a micro-reactor is limited for the following reasons: (1) the dielectric material separating cathode from anode is a source of contamination, (2) the incorporation of flow is difficult, and (3) device lifetime is adversely influenced by reactive gases. These issues have been addressed by replacing the dielectric with ambient gas and the planar cathode electrode with a metal capillary tube. The tube diameter $(d)$ must be selected so that the discharge can be struck in the hollow cathode mode at ambient pressure ( $p$ ), thus, the product $p d$ should be on the order of 10 Torr $\mathrm{cm}$ [4] For example, operation at one atmosphere requires that the tube diameter should be around $130 \mu \mathrm{m}$. Experience has shown that atmospheric operation in the hollow cathode mode occurs in tubes with diameters below approximately $200 \mu \mathrm{m}$. Literature reports on striking hollow cathode discharges in larger diameter tubes $(0.4-2.0 \mathrm{~mm})$ are consistent with operation at much lower pressures than atmospheric [13]. Both $\mathrm{dc}$ and $\mathrm{rf}$ power have been used [14, 15], the latter being more complicated by the need for a matching network. Atmospheric operation was achieved in one case but the discharge was found to form on the surface of the electrodes [16].

Operation in the micro-hollow cathode mode produces an intense plasma in the centre of the capillary tube. The plasma contains a high concentration of energetic electrons, which could generate reactive radicals by electron impact. When a gas is flown through the tube, radical species production occurs over very short length scale, which suggests potential for very fast gas-phase chemistry. While there is a realm of applications where short contact times could be beneficial [17] we limit



Figure 10. Schematic diagram of the set-up used for diamond-like carbon deposition; the micro-discharge forms at the tip of the capillary tube and expands toward the heated substrate.

ourselves here to an application of these micro-discharges in materials deposition.

Micro-discharges in flow geometry have been studied in a variety of gases using stainless steel capillary tubes (Varian, Inc.) as the cathode. The basic properties of these "plasma microjets' have been described previously [18]. For thin film deposition, the set-up consists of a tube (cathode) and a substrate (anode), as depicted in figure 10. This stagnation point flow geometry is applicable to many gas chemistries and substrates. As an example, we describe here the growth of diamond crystals and films using $\mathrm{CH}_{4} / \mathrm{H}_{2}$ gas mixtures.

Diamond growth experiments were conducted on heated polycrystalline molybdenum foils ( $99.98 \%$ purity). The Mo foil was etched in a liquid solution of $\mathrm{HF} / \mathrm{HNO}_{3} / \mathrm{HCl}$ and ultra-sonicated in an acetone bath prior to placement in the deposition chamber. No scratching by diamond powder was necessary to initiate growth. Before each experiment, the deposition chamber was pumped down, then back-filled to 200 Torr with a $\mathrm{CH}_{4} / \mathrm{H}_{2}$ mixture in the desired composition. Experiments were performed with active pressure control at a flow rate of $100 \mathrm{sccm} \mathrm{H}_{2}$ plus varying amounts of $\mathrm{CH}_{4}$ between 0.1 and $1 \mathrm{sccm}$. The substrates were heated resistively to $800^{\circ} \mathrm{C}$; the temperature was measured in situ using a $\mathrm{Pt} / \mathrm{Pt}-\mathrm{Rh}$ thermocouple. Since the gas flow cooled the substrate, it was important that the thermocouple was in contact with the substrate directly behind the impingement point. Discharges were struck with the capillary tube end $2 \mathrm{~mm}$ away from the substrate while maintaining constant current and voltage during the growth. After growing for 2-4h, the films were characterized by scanning electron microscopy (SEM), energy-dispersive spectroscopy (EDS), and micro-Raman spectroscopy (using a Renishaw M1000 Raman Spectrometer system with a $514.5 \mathrm{~nm}$ Ar laser).

For the pressures studied (100-500 Torr), the discharge was remarkably stable over a wide range of currents (5-20 mA) and could be run for hundreds of hours with no apparent damage to the tube. Representative emission spectra of $\mathrm{H}_{2}$ and $\mathrm{CH}_{4} / \mathrm{H}_{2}$ micro-discharges are shown in figure 11 . In pure $\mathrm{H}_{2}$, the strongest emissions are from atomic hydrogen Balmer lines at $486.1 \mathrm{~nm}$ and $656.3 \mathrm{~nm}$, noted as $\mathrm{H}_{\beta}$ and $\mathrm{H}_{\alpha}$, respectively. 


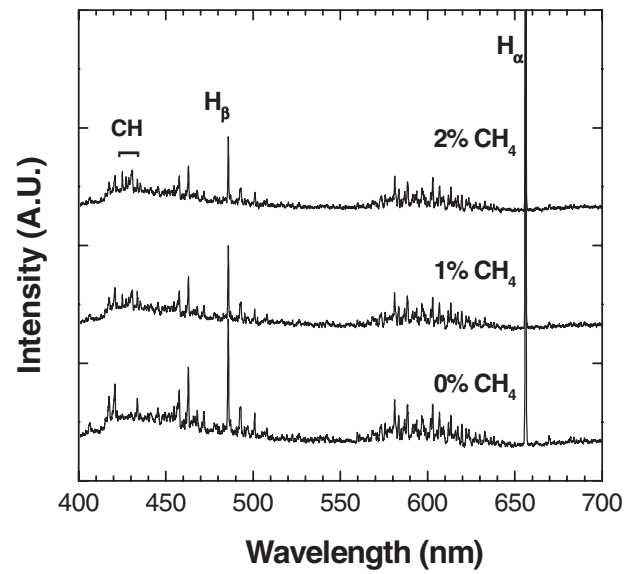

Figure 11. Optical emission spectra from $\mathrm{H}_{2}$ and $\mathrm{CH}_{4} / \mathrm{H}_{2}$ flowing micro-discharges operated at 200 Torr and $20 \mathrm{~mA}$. Note the presence of peaks corresponding to $\mathrm{CH}$ radicals when $\mathrm{CH}_{4}$ is supplied.

Many of the other lines in the spectrum are weaker atomic and molecular hydrogen states. When $\mathrm{CH}_{4}$ is added, lines appear in the region of $430 \mathrm{~nm}$ corresponding to a band of $\mathrm{CH}$ [19]. These lines become more intense with increasing $\mathrm{CH}_{4}$ concentration. No lines corresponding to $\mathrm{C}_{2}$ or $\mathrm{CH}^{+}$were observed.

A single micro-discharge was used to grow diamond films employing gas mixtures consisting of $\mathrm{H}_{2}$ and varying amounts of $\mathrm{CH}_{4}$ as indicated above ${ }^{2}$. The gases were mixed in a region upstream of the microjet. For discharge currents of $20 \mathrm{~mA}$, experiments could be run for several hours with the same tube. No contamination of the grown films could be detected (e.g. from erosion of the stainless steel tube due to sputtering or evaporation). SEM images showed that the growth rate and morphology of the deposited films depended strongly on the $\mathrm{CH}_{4}$ concentration in the gas mixture. For values over $1 \% \mathrm{CH}_{4}$ in hydrogen, mostly carbon was deposited and filamentary fibres were seen between the two electrodes. At $0.5 \% \mathrm{CH}_{4}$, a somewhat continuous film was deposited over a $500 \mu \mathrm{m}$ diameter area. The film consisted of particles in high-density near the centre and increasingly sparse coverage moving outwards. A close-up of the film (figure 12(a)) showed microscale roughness and some triangular faceting representative of diamond. As the $\mathrm{CH}_{4}$ concentration was reduced further to 0.25 and $0.1 \%$, individual crystals were observed with welldefined facets as shown in figures $12(b)$ and $(c)$, respectively. Due to a drop in the growth rate, the films exhibited sparser coverage with the heaviest concentration of particles at the stagnation point. This type of isolated particle growth has been reported for an atmospheric-pressure inductively-coupled plasma torch in a similar flow geometry [20]. Interestingly, there was little or no growth at the stagnation point in the latter study. This was attributed to lower concentrations of atomic hydrogen at the centre than near the edges of the substrate. The improved coverage at the centre in our experiments is consistent with the highest concentration of excited states expected along the centreline of the hollow cathode.

Micro-Raman spectroscopy was performed to distinguish diamond-like carbon $\left(\mathrm{sp}^{3}\right)$ from amorphous carbon $\left(\mathrm{sp}^{2}\right)$ for

2 Since $\mathrm{CH}_{4}$ is introduced in very small amounts $(0.1-1 \mathrm{sccm})$, the flow rate approximates its relative volumetric percentage.
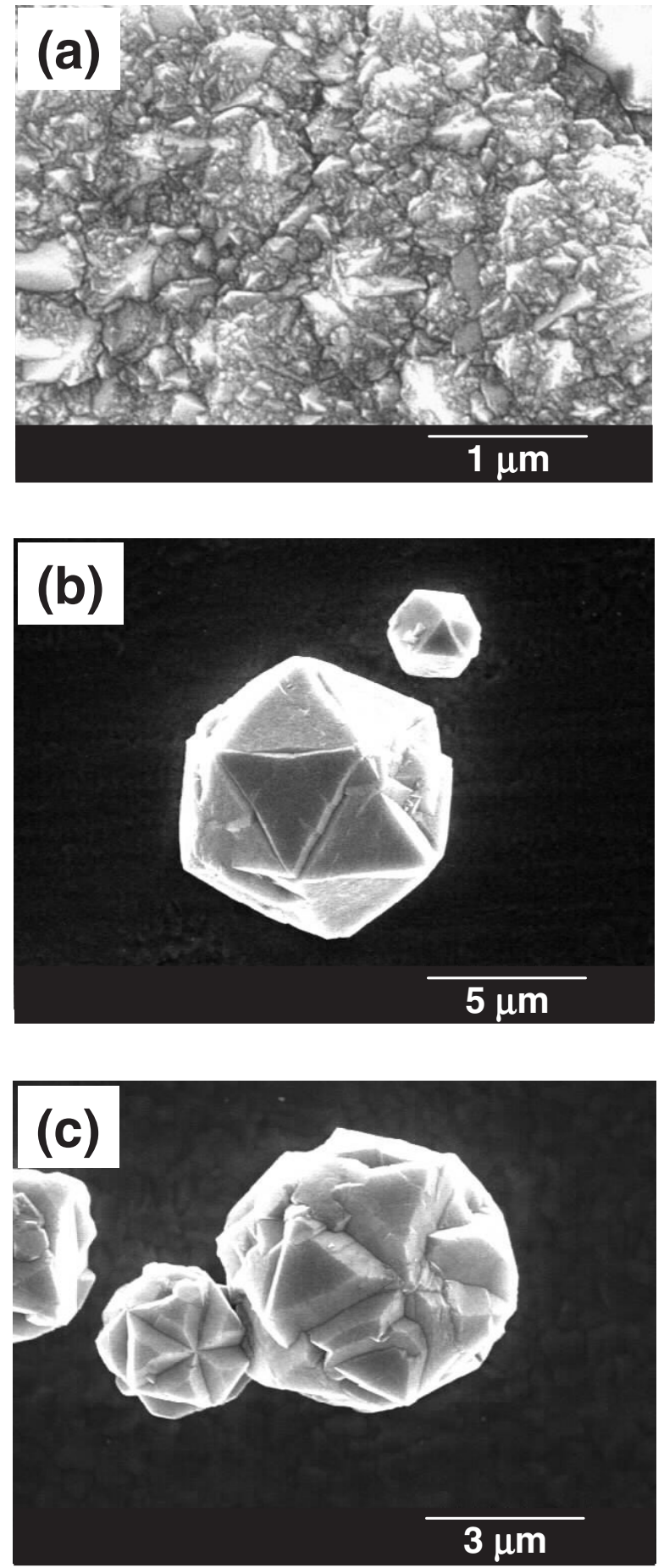

Figure 12. SEM images of diamond-like carbon grown using $\mathrm{CH}_{4} / \mathrm{H}_{2}$ flowing micro-discharges at 200 Torr for $2 \mathrm{~h}$ at methane concentrations of $(a) 0.5 \%,(b) 0.25 \%$, and (c) $0.1 \%$.

Cathode-anode gap $=2 \mathrm{~mm}$, total flow rate $=100 \mathrm{sccm}$, plasma current $=20 \mathrm{~mA}$, substrate temperature $=800{ }^{\circ} \mathrm{C}$.

each of the samples in figure 12 with the spectra shown in figure 13. The sharp peak that appears at approximately $1336 \mathrm{~cm}^{-1}$ is close to that of the first order optical phonon mode of natural diamond which occurs at $1332.5 \mathrm{~cm}^{-1}$. The shift in the peak is attributed to compressive stresses caused by lattice mismatch to a possible underlying $\mathrm{Mo}_{2} \mathrm{C}$ layer [20]. Broad peaks at 1350 and $1580 \mathrm{~cm}^{-1}$ attributed to $\mathrm{sp}^{2}$ 


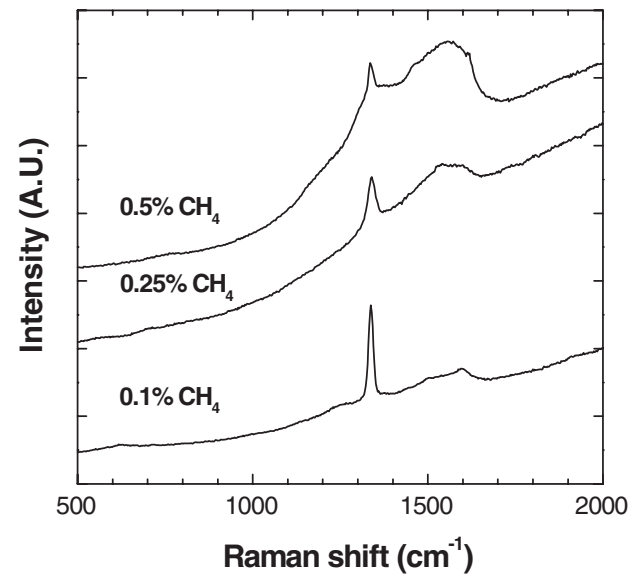

Figure 13. Micro-Raman spectra of diamond films corresponding to the diamond-like carbon samples shown in figure 12 .

carbon, were also present for films grown at the higher $\mathrm{CH}_{4}$ concentrations of $0.25 \%$ and $0.5 \%$. In the latter case, a shoulder at approximately $1150 \mathrm{~cm}^{-1}$ is also discernible which has been attributed to nano-crystals [21]. This observation is consistent with the smaller grain size seen in the SEM image of this film (figure 12(a)). As the $\mathrm{CH}_{4}$ concentration is reduced, the non-diamond peaks disappear and only a single peak at $1336 \mathrm{~cm}^{-1}$ remains (figure $12(c)$ ) suggesting a high purity micro-crystalline diamond phase.

At the optimum growth temperature of $800^{\circ} \mathrm{C}$, changes in pressure and flow rate were also studied to observe the effect on film morphology. An increase in the total flow rate to $200 \mathrm{sccm}$ at constant pressure (200 Torr) resulted in crystals exhibiting (100) faceting, in addition to the previously shown (111) faceting; compare figures $14(a)$ to $12(b)$. Some pitting and overgrowth on the crystal planes were also seen. Increasing the pressure to 400 Torr at constant total flow rate $(100 \mathrm{sccm})$, resulted in similar mixed faceting and more pitting. The reasons for the change in crystal morphology have not been investigated further.

The diamond growth experiments demonstrate that microdischarges can be used as a reactive source for depositing films of comparable quality to those produced by other often more complicated deposition techniques. The advantage of this tool is the simplicity of construction and operation, as well as the low power consumption. Thicker films are also possible since the source is stable and allows deposition of films continuously for much longer times. The coatings are restricted to small areas, but this may be desirable for materials processing on the micro-scale. Further, by using large arrays of tubes, micro-discharges could be utilized for the development of materials libraries for rapid property screening and optimization purposes.

\section{Summary}

In addition to lighting and spectroscopy applications, microdischarges operating at high-pressures offer advantages as micro-reactors for gas-phase chemistry or as miniaturized sources of ions and radicals. Two examples of their latter use in materials processing applications have been demonstrated.
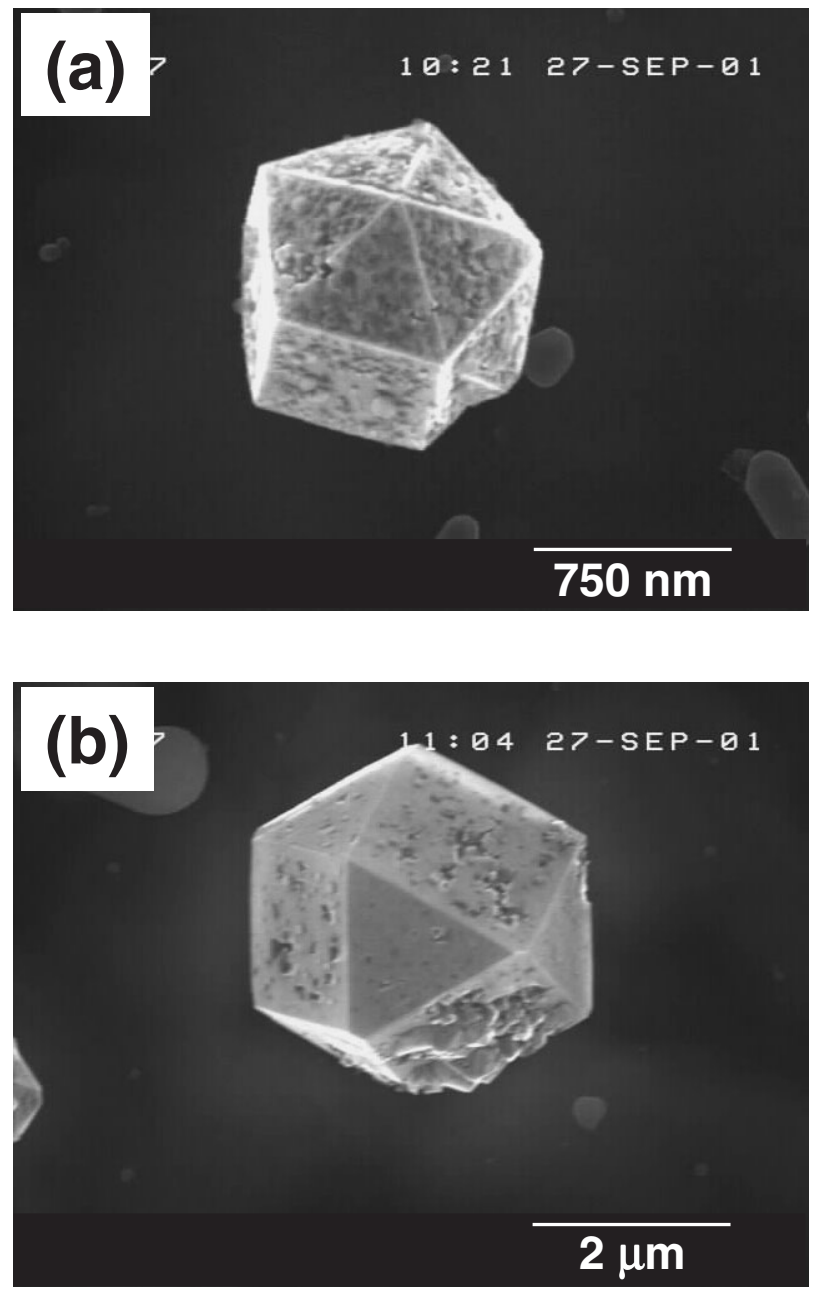

Figure 14. SEM images of diamond-like carbon crystals grown using $\mathrm{CH}_{4} / \mathrm{H}_{2}$ plasma microjets with $0.5 \%$ methane at (a) pressure $=200$ Torr, total flow rate $=200 \mathrm{sccm}$, and (b) pressure $=400$ Torr, total flow rate $=100 \mathrm{sccm}$.

Planar micro-discharges formed inside patterned cutouts on flexible metal-insulator-metal stencil masks have been used at relatively high-pressure (20 Torr) to directly pattern silicon wafers using $\mathrm{CF}_{4}$ and $\mathrm{SF}_{6}$ in Ar. Despite the low input power, etching occurs at high rates because the discharge forms only where it is needed at high density. Arrays of holes, lines, and combinations of them have been successfully patterned. The best results, in terms of high etch rate and low mask erosion, are obtained when the silicon substrate becomes part of the circuit, replacing the metal cathode of the layered structure. While the profile shape is initially anisotropic, it becomes self-limited due to expansion of the discharge into the etched cathode. Horizontal etching competes with vertical etching resulting in a bowl-shaped profile, which is different from that seen during isotropic etching and may be of interest for niche micro-fluidics applications.

Tubular (capillary) micro-discharges have been combined with flow of reactive precursors for diamond deposition. Diamond-like carbon crystals and films were deposited on molybdenum substrates at elevated temperatures using $\mathrm{CH}_{4} / \mathrm{H}_{2}$ chemistry. Characterization by micro-Raman spectroscopy demonstrated that the diamond-like carbon was of high quality, 
comparable to that obtained by other more complicated [10] Cho B, Ryu J, Hwang S, Lee G and Moon S H 2000 J. Vac. techniques. Sci. Technol. B 182769

[11] Park S J, Wagner C J, Herring C M and Eden J G 2000 Appl. Phys. Lett. 77199

\section{References}

[1] Schoenbach K H, Verhappen R, Tessnow T and Peterkin F E 1996 Appl. Phys. Lett. 6813

[2] Ngo M T, Schoenbach K H, Gerdin G A and Lee J H 1990 IEEE Trans. Plasma Sci. 18669

[3] Guenterschulze A 1923 Z. Phys. 19313

[4] Schoenbach K H, El-Habachi A, Shi W and Ciocca M 1997 Plasma Sources Sci. Technol. 6468

[5] Stark R H and Schoenbach K H 1999 Appl. Phys. Lett. 74 3770

[6] Frame J W, Wheeler D J, DeTemple T A and Eden J G 1997 Appl. Phys. Lett. 711165

[7] El-Habachi A and Schoenbach K H 1998 Appl. Phys. Lett. 7222

[8] El-Habachi A, Shi W, Moselhy M, Stark R H and Schoenbach K H 2000 J. Appl. Phys. 883220

[9] Park S J and Eden J G 2002 Appl. Phys. Lett. 814127

[12] White A D 1959 J. Appl. Phys. 30711

[13] Bardos L, Berg S and Blom H-O 1989 Appl. Phys. Lett. 55 1615

[14] Bardos L and Barankova H 2000 Surf. Coat. Technol. 133-134 522

[15] Brablec A, Kapicka V, Ondracek Z, Slavicek P, Strecha M, Stastny F and Vaculik R 1999 Czech. J. Phys. 49329

[16] Kapicka V, Sicha M, Klima M, Hubicka Z, Tous J, Brablec A, Slavicek P, Behnke J F, Tichy M and Vaculik R 1999 Plasma Sources Sci. Technol. 815

[17] Goetsch D A and Schmidt L D 1996 Science 2711560

[18] Sankaran R M and Giapis K P 2002 J. Appl. Phys. 922406

[19] Pearse R W B and Gaydon A G 1950 The Identification of Molecular Spectra (New York: Wiley) p 79

[20] Cappelli M A, Owano T G and Kruger C H 1990 J. Mater. Res. 52326

[21] Nemanich R J, Glass J T, Lucovsky G and Shroder R E 1988 J. Vac. Sci. Technol. A 61783 